\title{
La Biblioteca Apostolica Vaticana e il Papsturkundenwerk*
}

\author{
Don Raffaele Farina
}

Signor Presidente, cari colleghi e amici,

La mia presenza e la mia parola in questo importante Convegno Internazionale vogliono esprimere prima di tutto la cordiale adesione della Biblioteca Apostolica Vaticana, in particolare del Cardinale Bibliotecario, del Prefetto e degli Scriptores della Biblioteca, al centenario di Italia Pontificia e alla presente manifestazione scientifica; ma anche la solidarietà e la riconoscenza, in qualche modo anche a nome delle istituzioni scientifiche e culturali del Vaticano e della Santa Sede, alle istituzioni che hanno promosso e gestito nel secolo che ricordiamo una ricca attività scientifica e culturale universalmente apprezzata: mi riferisco ovviamente al Papsturkundenwerk e, in particolare, all'Accademia delle Scienze e all'Università di Göttingen, alla Pius-Stiftung e alle personalità che qui rappresentano tali benemerite istituzioni.

Presente, accanto all'Archivio Segreto Vaticano, pur se in maniera marginale e occasionale, sin dagli inizi, nel 1896, dell'attività del Papsturkundenwerk, la Biblioteca Apostolica Vaticana è entrata in maniera formale nel 1931, a norma dello Statuto $(\$ 10)$, tra i membri della Commissione Scientifica della PiusStiftung für Papsturkunden- und mittelalterlichen Geschichtsforschung. I rapporti e l'amicizia del fondatore Paul Fridolin Kehr con Achille Ratti, all'Ambrosiana prima e poi alla Vaticana, consolidatisi dopo l'elezione a Papa nel 1922, furono fondamentali per l'espansione e stabilizzazione del grande progetto scientifico del Papsturkundenwerk.

I contatti di Kehr erano soprattutto con il Cardinali Bibliotecario, il Card. Giovanni Mercati. In una lettera del 16 dicembre del 1936, Kehr esprime la sua preoccupazione per la salute del Papa: „la perdita di un padrone come Pio XI sarà per il piccolo ceto degli eruditi una vera catastrofe, perché la vecchia scienza non vale nel mondo civile di oggi più nulla". La descrizione del clima politico nella Berlino di quegli anni, la sua destituzione dalla direzione dei Monumenta Germaniae Historica e dell'Istituto Storico Prussiano, le difficoltà economiche non lo scoraggiano dal lavoro (a Merano trova un oasi di pace dover riesce a lavorare); e fa cenno, tra le altre pubblicazioni in preparazione, al IX volume dell'Italia Pontificia. Conclude esprimendo „la soddisfazione di una vita laboriosa, di cui il

\footnotetext{
* La corrispondenza citata in questo contributo è conservata nell'Archivio della Prefettura
} della Biblioteca Apostolica Vaticana tra i materiali e le carte non ancora inventariati. 
merito in gran parte ha il Santo Padre, il Card. Ehrle e Vostra Eminenza Rev.ma [il Card. Mercati]“. E il 17 ottobre dell'anno seguente, nel 1937, annuncia il suo viaggio a Roma: „ho ottenuto finalmente il permesso di partire per Roma coi materiali delle bolle pontificie (per qualche tempo sequestrati!!!), per finire i lavori da fare per l'edizione del IX volume dell'Italia pontificia“.

Alla fine della II guerra mondiale e nell'immediato periodo postbellico, la Santa Sede (in particolare il Sostituto alla Segreteria di Stato Mons. Giovanni Battista Montini), con la collaborazione del Cardinale Bibliotecario Card. Giovanni Mercati e del Prefetto P. Anselmo Albareda, è riuscita a rintracciare l'eredità di Paul Kehr, le sue carte, la fondazione in Svizzera. Ma queste sono vicende note, sulle quali è stato scritto. Il Card. Mercati, scrivendone il 15 gennaio 1947, al Presidente dell'Accademia delle Scienze, il Prof. Carl Friedrich Ruldolf Smend, ricorda che, per ordine di Pio XII, „fin dal gennaio del 1945, quando s'intese la morte del Kehr, la Segreteria di Stato ed io quale Cardinale Bibliotecario di S.R.C., non abbiamo cessato di ricercare, per tutte le vie che ci aprivano, tanto il fondo Pio XI per salvarlo dalla confisca e dalle distrazioni che ne potevano temere, e per assicurarne l'impiego totale e col maggior frutto nell'opera per cui era stato elargito, quanto per conoscere le disposizioni finali del Kehr, il quale negli ultimi colloqui con me si era mostrato non tranquillo sull'avvenire a causa delle mutate condizioni pubbliche e poi del sequestro per oltre mezzo anno dei materiali raccolti e delle sue carte, ed era dubbioso sul modo di provvedere efficacemente alla conservazione e all'uso di quelli e all'incolumità della fondazione".

L'anno precedente, nel 1946, il P. Anselmo Albareda, Prefetto della Biblioteca Vaticana, scrivendo al medesimo Presidente Smend, con quella diligenza e accuratezza che ne hanno fatto il migliore dei gestori della Biblioteca Vaticana nell'ultimo secolo, nota che „ora, dopo le pratiche più diligenti non è comparsa alcuna diversa disposizione del medesimo Kehr e quindi si può ritenere con pieno vigore lo Statuto del 23 novembre del 1931, sono stato avvertito di significarle la mia accettazione a membro della sopranominata Commissione e di compiere i doveri che ne discendono".

Cari colleghi e amici, nel concludere queste brevi annotazioni e citazioni, voglio esprimere qui pubblicamente la mia disponibilità e quella della Biblioteca Vaticana ad andare oltre gli obblighi che derivano dall'essere il Prefetto membro della Commissione Scientifica e a contribuire fattivamente allo sviluppo dell'attività della nostra Fondazione, della Pius-Stiftung, sull'esempio dei due grandi amici e fondatori, il Papa Achille Ratti Pio XI e il Prof. Paul Fridolin Kehr.

Vi ringrazio per la Vostra attenzione

Don Raffaele Farina 


\section{Zusammenfassung}

Der Autor überbringt den Tagungsteilnehmern den Gruß der Biblioteca Apostolica vaticana als deren Präfekt sowie ihr Repräsentant in der wissenschaftlichen Kommission der Pius-Stiftung für Papsturkunden- und mittelalterlichen Geschichtsforschung. Aus der unedierten Korrespondenz ergeben sich nicht nur die guten Beziehungen zwischen den beiden Institutionen, sondern vor allem die ausschlaggebende Hilfe, die der Pius-Stiftung vom Kardinalbibliothekar und vom Präfekt der Biblioteca vaticana sofort nach dem Krieg von 1945 an angeboten wurde, um den Geldbestand einzuholen und zu bewahren, der von Pius XI. in der Schweiz und vom Archiv der Pius-Stiftung gestiftet worden war, ebenso wie dokumentierende Güter und Urkunden, die Paul Fridolin Kehr hinterlassen hat. 
Bereitgestellt von | De Gruyter / TCS

Angemeldet | 217.89.40.26

Heruntergeladen am | 21.12.12 10:47 